\title{
Competition between Kondo Screening and Quantum Hall Edge Reconstruction
}

\author{
A. W. Heine, ${ }^{1, *}$ D. Tutuc, ${ }^{1}$ G. Zwicknagl, ${ }^{2}$ and R. J. Haug ${ }^{1}$ \\ ${ }^{1}$ Institut für Festkörperphysik, Leibniz, Universität Hannover, Appelstraße 2, 30167 Hannover, Germany \\ ${ }^{2}$ Institut für Mathematische Physik, Technische Universität Braunschweig, Mendelssohnstraße 3, 38106 Braunschweig, Germany
}

(Received 20 October 2015; published 4 March 2016)

\begin{abstract}
We report on a Kondo correlated quantum dot connected to two-dimensional leads where we demonstrate the renormalization of the $g$ factor in the pure Zeeman case. i.e., for magnetic fields parallel to the plane of the quantum dot. For the same system, we study the influence of orbital effects by investigating the quantum Hall regime; i.e., a perpendicular magnetic field is applied. In this case an unusual behavior of the suppression of the Kondo effect and of the split zero-bias anomaly is observed. The splitting decreases with magnetic field and shows discontinuous changes that are attributed to the intricate interplay between Kondo screening and the quantum Hall edge structure originating from electrostatic screening. This edge structure, made up of compressible and incompressible stripes, strongly affects the Kondo temperature of the quantum dot and thereby influences the renormalized $g$ factor.
\end{abstract}

DOI: 10.1103/PhysRevLett.116.096802

The Kondo effect [1] is one of the most fascinating many-body correlation effects. It was first discovered in metals with magnetic impurities and was attributed to the screening of these local magnetic moments by conduction electrons. In Coulomb-blockaded quantum dots (QDs) acting as highly tunable single magnetic impurities the Kondo effect manifests itself as a zero-bias anomaly (ZBA) in the enhanced conductance [2-6]. ZBAs appear quite often in transport experiments as a result of interaction effects; e.g., the claim of the observation of Majorana fermions was related to a ZBA [7]. The origin of such a ZBA can be identified by the detailed understanding of the influence of magnetic fields, as in the case of the Majorana fermions. But the influence of magnetic fields on interaction and correlation effects can be quite intricate. In general, the ZBA of the Kondo effect is suppressed by a sufficiently high magnetic field. The accepted physical picture is that an external magnetic field polarizes the magnetic impurity removing the spin degeneracy, which is the prerequisite for the appearance of the small energy scale. The low-energy excitations giving rise to the Kondo resonance can be described in terms of fermionic strongly renormalized "heavy" spin- $1 / 2$ quasiparticles. In a magnetic field the energies of spin-1/2 quasiparticles with spin $\sigma= \pm$ will be shifted by the Zeeman energy, $\tilde{b}_{\sigma}=$ $\frac{1}{2} \tilde{g} \sigma \mu_{B} B$, where $B$ and $\mu_{B}$ are the magnetic field and the Bohr magneton, respectively. The effective $g$ factor $\tilde{g}$, whose bare value is $g=0.44$ for GaAs, is renormalized by the Kondo effect $[8,9]$. It assumes twice its bare value in the limit of low external fields as predicted by Wilson [10]. The central focus of the present study is the evolution of the Zeeman splitting with magnetic field. The system under consideration is a Kondo-correlated QD connected to twodimensional leads. Of particular interest is a discontinuous change in the Zeeman splitting of the ZBA observed upon tuning the filling factor of the leads in the quantum Hall regime through an integer value. Our conjecture is that the abrupt changes reflect the reconstruction of the quantum Hall edge sketched schematically in Fig. 1.

The experimental data were obtained on a QD fabricated in a two-dimensional electron gas (2DEG) $37 \mathrm{~nm}$ below the surface. The electron density of $n_{e}=3.95 \times 10^{15} \mathrm{~m}^{-2}$ and the electron mobility of $\mu_{e}=65.6 \mathrm{~m}^{2} / \mathrm{Vs}$ of the 2DEG were determined at liquid helium temperature. A QD is formed by oxide lines produced by local anodic oxidation $[11,12]$. Its diameter is about $140 \mathrm{~nm}$ and it is coupled to source $(S)$ and drain $(D)$ via tunnel barriers. Three in-plane gates $(G 1, G 2$, and $G 3)$ are used to control the tunnel coupling to the leads and the energy levels on the dot [13]. An atomic force microscope (AFM) image of the sample is shown in Fig. 2(a). The measurements were performed in a ${ }^{3} \mathrm{He} /{ }^{4} \mathrm{He}$ dilution refrigerator with a base temperature of $20 \mathrm{mK}$ using standard lock-in technique. Coulomb blockade measurements revealed the QD's charging energy of $U=350 \pm 30 \mu \mathrm{eV}$. The number of electrons confined in the QD is estimated to be $61 \pm 3$.

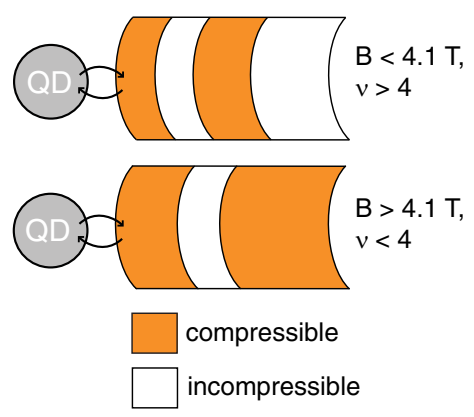

FIG. 1. Sketch of the edge channel structure near the QD for noninteger filling factors immediately above $\nu=4$ (top) and below $\nu=4$ (bottom), respectively. 

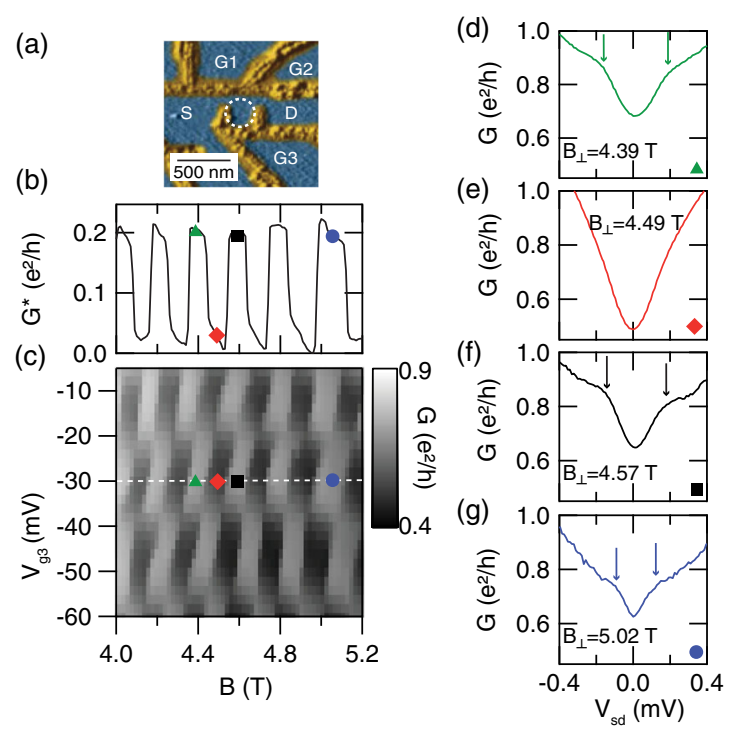

FIG. 2. (a) An AFM image of the sample. The oxide lines that define the structure are marked in yellow. (b) Difference $G^{*}$ in the differential conductance between Kondo enhanced and Coulomb blocked regions as a function of magnetic field along the dashed line in (c). The background was subtracted. (c) Differential conductance through the QD as a function of perpendicular magnetic field $B_{\perp}$ and gate voltage $V_{G 3}$. (d)-(g) Bias dependence of $G$ for fixed values of $B_{\perp}$ referring to points in (b) and (c), which are marked by respective symbols. Shoulders are indicated by arrows.

Magnetic fields applied perpendicular to the 2DEG affect spin and orbital motion of the electrons leading to the formation of Landau levels in the QD and in the leads. For a multielectron QD a complicated level structure with many occupied Landau levels appears at small and intermediate magnetic fields. At large enough magnetic fields with only two Landau levels occupied, the situation simplifies. For the Kondo effect only the outermost states of the QD belonging to the lowest Landau level (LL0) participate in Kondo transport [14-16]. An even or odd number of electrons on LLO is determining whether Kondo enhanced transport is possible. Switching between even and odd occupation of LLO is achieved either by redistribution of electrons between the two lowest Landau levels due to magnetic field modulation or by the loading of additional electrons to the dot by varying the gate voltage. The resulting pattern is often called the Kondo chessboard. A typical example is shown in Fig. 2(c) for our sample. The differential conductance $G=d I / d U_{s d}$ of the QD as a function of perpendicular magnetic field $B_{\perp}$ and gate voltage is depicted there. At magnetic field values between 4 and $5 \mathrm{~T}$, a regular pattern of alternating tiles of high and low conductance is visible. Low conductance corresponds to Coulomb blockade and high conductance to Kondo enhanced transport due to an odd number of electrons in LL0. The modulation caused by the magnetic field is shown in Fig. 2(b) for a fixed gate voltage. At every step in the conductance a flux quantum is added to the QD and the electron number in LL0 is changed by one [15].

Measuring $G$ as a function of bias in various regions of this Kondo chessboard leads to the results seen in Figs. 2(d)-2(g). In tiles without Kondo transport [Fig. 2(e)], a minimum can be observed at zero bias, as expected for a Coulomb-blocked QD. In contrast, a higher conductance at zero bias and two shoulders at finite bias can be distinguished in Figs. 2(d),2(f), and 2(g) for the Kondo regime. These shoulders indicate a splitting of the ZBA. Comparing the splitting at $4.39 \mathrm{~T}$ (green triangle) and $4.57 \mathrm{~T}$ (black square) yields the following observation: the splitting at $4.39 \mathrm{~T}, \Delta V_{s d}=0.38 \pm 0.02 \mathrm{mV}$, is slightly larger than the one at $4.57 \mathrm{~T}, \Delta V_{s d}=0.32 \pm 0.01 \mathrm{mV}$, a result that cannot be explained by bare Zeeman splitting $\Delta_{Z}=g \mu_{B} B$. Comparing the splitting at higher $B_{\perp}=5.02 \mathrm{~T}$ (blue circle) shows an even narrower splitting of $\Delta V_{s d}=0.21 \pm$ $0.02 \mathrm{mV}$. To further examine the development of the splitting it was analyzed for three successive electron numbers, which is possible due to the chessboard pattern. A quantitative examination of the splitting width [17] in units of $\Delta_{Z}$ is shown in Fig. 3(a). Quite astonishingly, the splitting decreases with increasing magnetic field and shows a discontinuity at $B_{\perp}=4.1 \mathrm{~T}$. In the following investigation of the underlying physics, we focus on the discontinuity around $B=4.1 \mathrm{~T}$, which we shall attribute to the quantum Hall effect in the "metallic" leads since it does not depend upon the number of electrons on the QD and it occurs at a magnetic field value where an integer filling
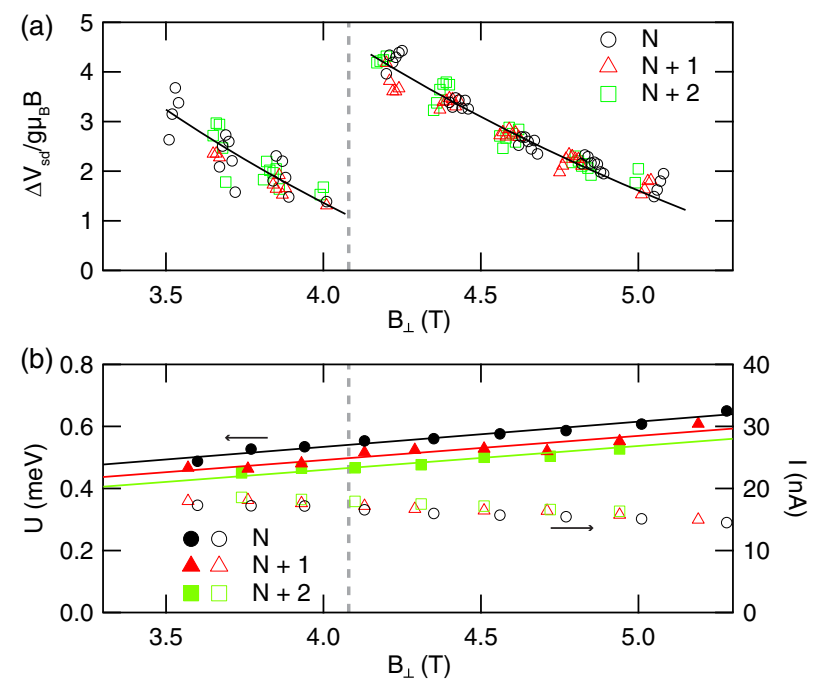

FIG. 3. (a) Evolution of the splitting width in the Kondo chessboard for three successive electron numbers in the range of $N \approx 60$. The dashed line indicates the integer filling factor $\nu=4$ in the leads. (b) Charging energy $U$ and current $I$ at fixed source drain voltage $V_{s d}=0.5 \mathrm{mV}$ in non-Kondo regions as a function of magnetic field for the same electron numbers as shown in (a). Filled symbols show $U$ (left axis), open symbols show $I$ (right axis). The solid lines are a guide to the eye to show the linear increase of $U$. 
factor $\nu$ appears in the leads for the given electron concentration $n_{e}=3.95 \times 10^{15} \mathrm{~m}^{-2}$. The step in the splitting width is much larger than expected for the influence of magnetic field modulated Fermi energy on the Zeeman splitting due to nonparabolicity of the conductance band [18].

Furthermore, the step in the splitting width is not a result of a change in the coupling strength $\Gamma$ of the QD to the leads nor a change of the charging energy of the QD. The behavior of $\Gamma$, which can be derived from the current $I$ through the QD, decreases smoothly with increasing magnetic field, as can be seen from Fig. 3(b) for a fixed $V_{s d}=0.5 \mathrm{mV}$. The evolution of $U$ was estimated by fitting the linear asymptotes of the current-voltage characteristic and determining the difference between these fits on both sides. The difference of the asymptotes is $U / 2[19,20]$. Both $I$ and $U$ vary only slowly with magnetic field and exhibit no particular behavior around $4.1 \mathrm{~T}$, as can be seen in Fig. 3(b). The increase of $U$ and the decrease of $\Gamma$ with magnetic field are easily explained by compression of the electron wave functions on the QD and in the leads leading to a slightly lower capacitance, i.e., a higher charging energy.

Before we discuss the influence of the perpendicular magnetic field on the splitting width, we take a look at the Zeeman splitting of the ZBA in a parallel magnetic field. Application of magnetic fields in parallel to the sample surface $\left(B_{\|}\right)$affects only the spins of the electrons due to the strong confinement of the 2DEG, whereas application of a magnetic field perpendicular to the sample surface $\left(B_{\perp}\right)$ and as such also perpendicular to the 2DEG also influences the orbital motion of the electrons. Figure 4(a) shows typical measurements of the differential conductance $G=d I / d V_{s d}$ as a function of bias for different magnetic fields. At $B=0$ the Kondo resonance at zero bias is visible as a ZBA. The half width at half maximum $\Delta_{\mathrm{ZBA}}=50 \pm$ $9 \mu \mathrm{eV}$ of this resonance is determined using a Lorentzian fit. The Kondo temperature $T_{K}=188 \pm 34 \mathrm{mK}$ is estimated from $T_{K}=\pi w \Delta_{\mathrm{ZBA}} / 4 k_{B}$, with $w=0.4128$ being the Wilson number [1]. The ZBA does not change for small values of $B_{\|}$, as can be seen at $B_{\|}=0.2 \mathrm{~T}$. This insensitivity towards application of magnetic fields is expected as long as the width of the ZBA given by the Kondo temperature is larger than the Zeeman splitting. For magnetic fields exceeding a threshold value of $B_{t}=0.5 \pm 0.1 \mathrm{~T}$, the peak starts to shift, indicating a Zeeman splitting of the resonance [27-29]. Considering the $g$ factor for bulk GaAs, $g=0.44$, our threshold value yields a bare Zeeman splitting of $g \mu_{B} B_{t}=13 \pm 3 \mu \mathrm{eV}$, which is of the same order of magnitude as the value for $\Delta_{\mathrm{ZBA}}$ obtained at $B_{\|}=0$. Comparing the bare Zeeman splitting at the threshold to the width of the ZBA, however, we are led to the conclusion that the actual splitting must be enhanced over its bare counterpart. A typical trace for the split Kondo peak is displayed for $B_{\|}=2 \mathrm{~T}$ in Fig. 4(a).
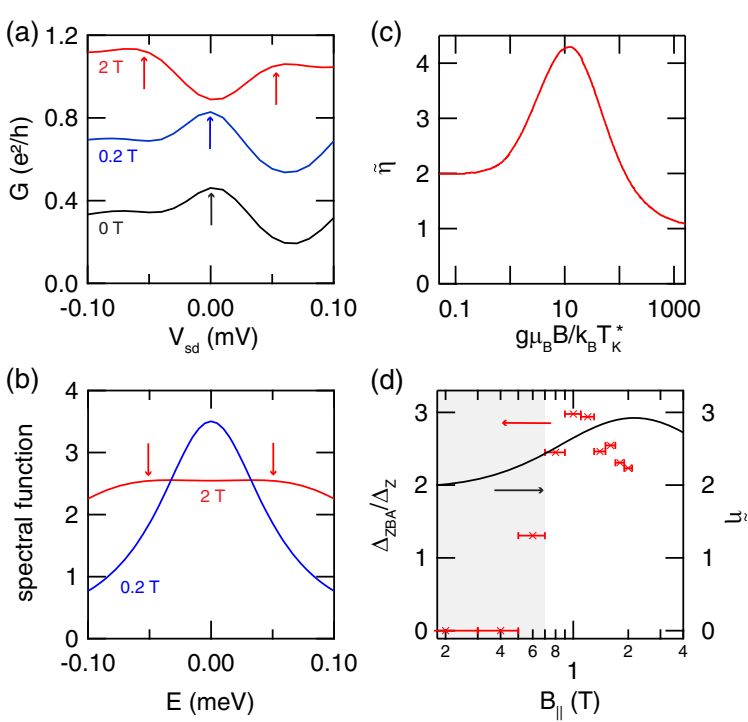

FIG. 4. (a) Differential conductance through the QD as a function of bias. An offset of 0.35 and $0.7 e^{2} / h$, respectively, is added to the traces at 0.2 and $2 \mathrm{~T}$ for clarity. The Kondo resonance at 0 and $0.2 \mathrm{~T}$ and the symmetric peaks at $2 \mathrm{~T}$ are marked by arrows. (b) Calculation of the spectral function as a function of energy for two different magnetic field values. The arrows point out the maxima of the function. (c) Enhancement factor $\tilde{\eta}$ over $g \mu_{B} B / k_{B} T_{K}^{*}$ on a logarithmic scale with $T_{K}^{*}=T_{K} / w$ being the Kondo temperature at $T=0$. The presented data were taken from Ref. [8]. (d) Red crosses show the normalized splitting of the ZBA, $\Delta_{\mathrm{ZBA}}$, as a function of parallel magnetic field. The black line represents results for the enhancement factor $\tilde{\eta}$ derived from renormalized perturbation calculations. In the gray area the splitting is suppressed and the theoretical data do not apply.

This can also be seen in Fig. 4(b) where results for calculated spectral functions at 0.2 and $2 \mathrm{~T}$, respectively, are shown: at $0.2 \mathrm{~T}$ there is still only a single peak at zero bias while at $2 \mathrm{~T}$ a sum of two Lorentz functions centered at $\pm 0.75 \mathrm{meV}$ is obtained.

While the Anderson model for a correlated impurity with spin $S=1 / 2$ certainly has its limitations in describing the complex electronic structure of a QD with 60 electrons, it nevertheless provides a semiquantitative explanation of the observed phenomena. For simplicity we assume particlehole symmetry. In the limit $U \gg \pi \Gamma$, the model calculations shown in Fig. 4(c) predict a nonmonotonic variation of the enhancement factor $\tilde{\eta}=\tilde{g} / g$ from the low-field value $\tilde{\eta}=2$ to $\tilde{\eta}=1$ at high fields going through a maximum [8]. The existence of the latter can be easily understood from the competition between formation of a local moment and transversal spin fluctuations. This enhancement can be observed in the experimental results for $\Delta V_{s d}$ as obtained from the measurements that are shown in Fig. 4(d). The quantitative deviations from the universal strong-coupling behavior should be ascribed to the fact that the system under consideration is rather in the intermediate coupling regime. In fact, the relatively large value $\Delta_{\mathrm{ZBA}} / U$ suggests 
$U / \pi \Gamma=1.26$. We adopt the renormalized perturbation theory [9] to calculate the variation with magnetic field of the enhanced Zeeman splitting [30]. The results for $U=350 \mu \mathrm{eV}$ and $\Gamma=57 \mu \mathrm{eV}$ displayed in Fig. 4(d) reproduce the experimental data qualitatively. The parameters chosen underestimate the width of the Kondo resonance by a factor of 3 . This discrepancy can be explained by the fact that we reduced the electronic structure of the QD to a single spin-degenerate level. It is well known that, for a multilevel QD, $T_{K}$ is enhanced [31].

Having explained the evolution with magnetic field $B_{\|}$of the Kondo resonance, we next turn to the fields $B_{\perp}$ applied perpendicular to the QD and present one possible interpretation of the observations in Fig. 3. Assuming the different splitting width in the perpendicular field to be a consequence of a discontinuous variation of $\tilde{g}$ leads to a sharp change in $T_{K}$ since $B_{\perp}$ is quasiconstant at this point. However, the change in $T_{K}$ is not the result of a change in the coupling between the QD and the leads or a variation of $U$ as shown above. The key to an understanding of the findings above is that (i) the Kondo effect is essentially a screening process and (ii) the screening properties of a 2DEG may depend sensitively on the filling factor of the Landau levels in the quantum Hall regime. To understand the interplay of these two phenomena, we have to compare their characteristic length scales. The prevailing view of the Kondo effect is that at low temperatures $T \ll T_{K}$ the magnetic moment of the QD forms a singlet with the conduction electrons. The magnetic impurity becomes invisible outside a screening cloud [32,33] of spatial extent $\xi_{K}=\hbar v_{F} / k_{B} T_{K}$, with $v_{F}$ being the Fermi velocity in a two-dimensional system and $\hbar$ the Planck constant. Since $k_{B} T_{K}$ is comparable to the width of the Kondo resonance $\Delta_{\mathrm{ZBA}}$, one can write $\xi_{K}=\hbar v_{F} / \Delta_{\mathrm{ZBA}}$.

The formation of the Kondo cloud reflects the screening properties of the leads. The latter, however, are known to change dramatically with magnetic field in the quantum Hall regime. It is generally accepted that the magnetic field acting on the orbital motion of the conduction electrons leads to the formation of compressible regions that are separated by incompressible strips. The characteristic length scale $l_{B}$ for the edge channels is set by electrostatics and is, as such, only weakly affected by external magnetic fields [34]. Simple estimates show that $l_{B} \approx 100$ $\ll \xi_{K} \approx 5000 \mathrm{~nm}$. As a consequence, the Kondo effect will be affected by changes in the edge structure and also by changes in the interior of the leads, since both of them will lead to an additional confinement of the Kondo cloud [35]. Screening is possible only from the compressible metallic regions. As a result, we anticipate a (partial) suppression of the Kondo effect when large parts of the leads become incompressible. This suppression happens indeed whenever the bulk filling factor approaches an integer value from above [36,37], as illustrated in Fig. 1. In this field regime compressible regions exist only close to the edges and screening originates almost exclusively from edge channels. For filling factors slightly below an integer value, on the other hand, large portions of the leads are compressible and should be available for screening leading to a rise in the Kondo temperature. Away from an integer $\nu$, the incompressible regions grow with increasing $B$, as can clearly be seen in Ref. [37]. This leads to a monotonic decrease in $T_{K}$ and therefore to a decrease of the renormalized Zeeman splitting. Thus, the unusual decline of the splitting width can be explained.

In summary, we measured the Zeeman splitting of the ZBA in parallel and perpendicular magnetic field. While in parallel fields the splitting increases nonmonotonically with magnetic field, as expected from the renormalized $g$ factor. In the so-called Kondo chessboard, the splitting of the Kondo resonance decreases with increasing magnetic field and shows a sharp discontinuity. This behavior shows the dependence of the renormalized Zeeman splitting of the Kondo resonance on the edge reconstruction due to the quantum Hall effect. The screening properties of the twodimensional leads depend on the distribution of compressible states contributing to screening and incompressible states which do not participate in Kondo screening.

We thank B. Popescu for producing the sample and we thank B. Shklovskii and A. C. Hewson for discussions. We acknowledge funding by the School for Contacts in Nanosystems.

*heine@ nano.uni-hannover.de

[1] A. C. Hewson, The Kondo Problem to Heavy Fermions (Cambridge University Press, Cambridge, England, 1993).

[2] D. Goldhaber-Gordon, H. Shtrikman, D. Mahalu, D. Abusch-Magder, U. Meirav, and M. A. Kastner, Nature (London) 391, 156 (1998).

[3] D. Goldhaber-Gordon, J. Göres, M. A. Kastner, H. Shtrikman, D. Mahalu, and U. Meirav, Phys. Rev. Lett. 81, 5225 (1998).

[4] S. M. Cronenwett, T. H. Oosterkamp, and L. P. Kouwenhoven, Science 281, 540 (1998).

[5] J. Schmid, J. Weis, K. Eberl, and K. v. Klitzing, Physica (Amsterdam) 256-258B, 182 (1998).

[6] W. G. van der Wiel, S. De Franceschi, T. Fujisawa, J. M. Elzerman, S. Tarucha, and L. P. Kouwenhoven, Science 289 , 2105 (2000).

[7] V. Mourik, K. Zou, S. M. Frolov, S. R. Plissard, E. P. A. M. Bakkers, and L.P. Kouwenhoven, Science 336, 1003 (2012).

[8] A. C. Hewson, J. Bauer, and W. Koller, Phys. Rev. B 73, 045117 (2006).

[9] K. Edwards and A. C. Hewson, J. Phys. Condens. Matter 23, 045601 (2011).

[10] K. G. Wilson, Rev. Mod. Phys. 47, 773 (1975).

[11] R. Held, T. Vancura, T. Heinzel, K. Ensslin, M. Holland, and W. Wegscheider, Appl. Phys. Lett. 73, 262 (1998).

[12] U. F. Keyser, H. W. Schumacher, U. Zeitler, R. J. Haug, and K. Eberl, Appl. Phys. Lett. 76, 457 (2000). 
[13] D. Tutuc, B. Popescu, D. Schuh, W. Wegscheider, and R. J. Haug, Phys. Rev. B 83, 241308 (2011).

[14] M. Keller, U. Wilhelm, J. Schmid, J. Weis, K. v. Klitzing, and K. Eberl, Phys. Rev. B 64, 033302 (2001).

[15] C. Fühner, U. F. Keyser, R. J. Haug, D. Reuter, and A. D. Wieck, Phys. Rev. B 66, 161305 (2002).

[16] M. Stopa, W. G. van der Wiel, S. De Franceschi, S. Tarucha, and L.P. Kouwenhoven, Phys. Rev. Lett. 91, 046601 (2003).

[17] To determine the position of the shoulders in $G$, they were fitted with a Gaussian function and an additional cubic background.

[18] M. Dobers, K. v. Klitzing, and G. Weimann, Phys. Rev. B 38, 5453 (1988).

[19] P. J. M. van Bentum, H. van Kempen, L. E. C. van de Leemput, and P. A. A. Teunissen, Phys. Rev. Lett. 60, 369 (1988).

[20] See Supplemental Material at http://link.aps.org/ supplemental/10.1103/PhysRevLett.116.096802, which includes Refs. [21-26], for more details on the extraction of $U$.

[21] P. W. Anderson, Phys. Rev. 124, 41 (1961).

[22] A. C. Hewson, A. Oguri, and D. Meyer, Eur. Phys. J. B 40, 177 (2004).

[23] A. C. Hewson, J. Phys. Condens. Matter 13, 10011 (2001).

[24] E. Peskin and D. V. Schroeder, An Introduction to Quantum Field Theory (Westview Press, Reading, MA, 1995).

[25] Mark Srednicki, Quantum Field Theory (Cambridge University Press, Cambridge, England, 2007).
[26] K. Edwards, A. C. Hewson, and V. Pandis, Phys. Rev. B 87, 165128 (2013).

[27] A. Kogan, S. Amasha, D. Goldhaber-Gordon, G. Granger, M. A. Kastner, and H. Shtrikman, Phys. Rev. Lett. 93, 166602 (2004).

[28] S. Amasha, I. J. Gelfand, M. A. Kastner, and A. Kogan, Phys. Rev. B 72, 045308 (2005).

[29] C. H. L. Quay, J. Cumings, S. J. Gamble, R. de Picciotto, H. Kataura, and D. Goldhaber-Gordon, Phys. Rev. B 76, 245311 (2007).

[30] See Supplemental Material at http://link.aps.org/ supplemental/10.1103/PhysRevLett.116.096802 for a description of this method.

[31] I. Garate and I. Affleck, Phys. Rev. Lett. 106, 156803 (2011).

[32] H. Prüser, M. Wenderoth, P. E. Dargel, A. Weismann, R. Peters, T. Pruschke, and R. G. Ulbrich, Nat. Phys. 7, 203 (2011).

[33] A. K. Mitchell, M. Becker, and R. Bulla, Phys. Rev. B 84, 115120 (2011).

[34] D. B. Chklovskii, B. I. Shklovskii, and L. I. Glazman, Phys. Rev. B 46, 4026 (1992).

[35] Perspectives of Mesoscopic Physics - Dedicated to Prof Yoseph Imry's 70th Birthday, edited by A. Aharony and O. Entin-Wohlmann (World Scientific Publishing, Singapore, 2010).

[36] N. B. Zhitenev, R. J. Haug, K. v. Klitzing, and K. Eberl, Phys. Rev. Lett. 71, 2292 (1993).

[37] A. Siddiki and R. R. Gerhardts, Phys. Rev. B 70, 195335 (2004). 